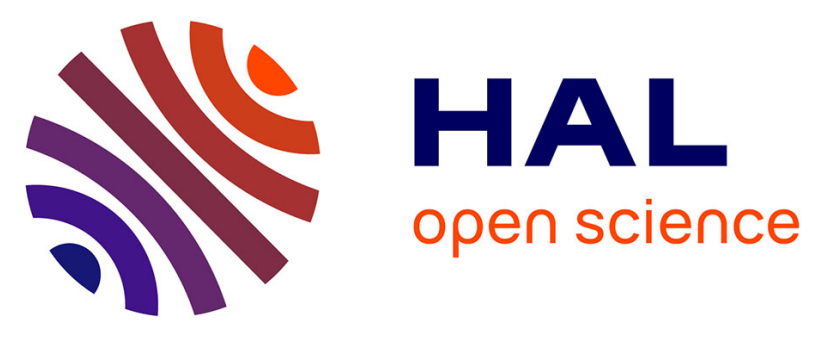

\title{
Real-Time Dynamic Power Management based on Pearson's Correlation Coefficient
}

Arthur de Miranda Neto, Alessandro Correa Victorino, Isabelle Fantoni, Douglas Eduardo Zampieri

\section{To cite this version:}

Arthur de Miranda Neto, Alessandro Correa Victorino, Isabelle Fantoni, Douglas Eduardo Zampieri. Real-Time Dynamic Power Management based on Pearson's Correlation Coefficient. 15th International Conference on Advanced Robotics (ICAR 2011), Jun 2011, Tallinn, Estonia. pp.304-309. hal00657242

\section{HAL Id: hal-00657242 https://hal.science/hal-00657242}

Submitted on 6 Jan 2012

HAL is a multi-disciplinary open access archive for the deposit and dissemination of scientific research documents, whether they are published or not. The documents may come from teaching and research institutions in France or abroad, or from public or private research centers.
L'archive ouverte pluridisciplinaire HAL, est destinée au dépôt et à la diffusion de documents scientifiques de niveau recherche, publiés ou non, émanant des établissements d'enseignement et de recherche français ou étrangers, des laboratoires publics ou privés. 


\title{
Real-Time Dynamic Power Management based on Pearson's Correlation Coefficient
}

\author{
A. Miranda Neto, A. Corrêa Victorino, I. Fantoni and D. E. Zampieri
}

\begin{abstract}
An autonomous robotic platform should be able to perform long-range and long-endurance missions, which energy limitation is one of the most important challenges. Studies show that motion is not the only power consumer. Management of all power resources is therefore important for these systems. Moreover, many applications for control of autonomous platform are being developed and one important aspect is the excess of information, frequently redundant, that imposes a great computational cost in data processing and consequently power consummation. In our previous works, we have proposed a visual-perception system based on an automatic image discarding method as a simple solution to improve the performance of a real-time navigation system. In this paper, we propose a new environment observer method based on Pearson's Correlation Coefficient. This monocular-vision system permits that some logical components may be shut down to save processor energy consumption, and/or to make the CPU available for running concurrent processes. Nevertheless, this method may be extended to other sensors and components. Our real-time perception system has been evaluated from real data obtained by our intelligent vehicle. It is not based on previous knowledge of the environment neither camera calibration.
\end{abstract}

\section{INTRODUCTION}

$\mathrm{S}$ everal applications for control of autonomous and semiautonomous platforms are being developed. The challenge to construct robust methods, and, in most cases, optimized systems, remains an open problem. Some of these applications include: aerial robots [1], [2], Grand Challenge [3], Urban Challenge [4] and Advanced Driver Assistance Systems (ADAS) [5].

The aerial robots offer great perspectives in many applications: search and rescue, outdoor and indoor building inspection, real-time monitoring, high risk aerial missions, mapping, fire detection or cinema recording [1], [2]. Similarly, the development of Unmanned Aerial Vehicles (UAVs) has been of interest for military applications, and the focus has been placed on creating small platforms. These vehicles are primarily used for surveillance and reconnaissance, however, one limitation is their maximum

Manuscript received May 24, 2011.

Arthur de Miranda Neto is Ph.D. Student in Mechanical Engineering at the State University of Campinas - Brazil and in Information Technology and Systems at the University of Technology of Compiègne, France. Actually, he is researcher engineer at the Heudiasyc Lab CNRS UMR 6599.

Alessandro Corrêa Victorino is associate professor at the University of Technology of Compiègne - Heudiasyc Lab CNRS UMR 6599, France.

Isabelle Fantoni is researcher at the University of Technology of Compiègne - Heudiasyc Lab CNRS UMR 6599, France.

Douglas Eduardo Zampieri is associate professor of the Computational Mechanics Department, at the State University of Campinas, Brazil. flight time, therefore they cannot carry large fuel payloads [6]. Future exploration of Mars, laid out by the Vision for Space Exploration, requires long-endurance UAVs that use resources that are plentiful on Mars [7], [8]. Including reactive intelligence based on an autonomic sense-act modality and sensed by optical image processing, the purpose of the Energetically Autonomous Tactical Robot (EATR) ${ }^{\mathrm{TM}}$ project is to develop an autonomous robotic platform able to perform long-range, long-endurance missions without the need for manual or conventional refueling [9], [10]. Finally, for military or civil purposes, vehicular applications [3], [4], [5] have as objective the development of autonomous and semi-autonomous systems capable of traversing unrehearsed and off-road terrain, driving a car autonomously in an urban environment and also to help the driver in its driver task.

These real-time intelligent platform developments have a common issue: providing to the platform the capability of perceiving and interacting with its neighbour environment, managing power consumption, the CPU usage, etc. These problems of time-dependent and dynamic resource allocation have manifested themselves under different names in application domains such as embedded systems and digital circuits, which include energy and memory consumption for the embedded systems [11]. It has been a topic of interest in the automotive industry [12], [13], [14].

In this paper, a real-time perception problem is applied to intelligent vehicles (human operated or autonomous systems). We propose a new environment observer method based on Pearson's Correlation Coefficient [15]. This monocular-vision system observes if there are no significant changes in the environment, permitting that some logical components may be shut down to save processor energy consumption, and/or to make the CPU available for running concurrent processes.

In Section II we present a review of previous works. Section III and IV introduce the Pearson's Correlation Coefficient and the Discarding Criteria. The Logical Dynamic Optimization methodology and results are presented in the Section V and VI. The conclusions are given in Section VII.

\section{RELATED WORKS}

Autonomous robots can perform desired tasks in unstructured environments without continuous human guidance. These systems have some degree of self- 
sufficiency. Self-configuring, self-optimizing and selfprotecting are still an open question. For advances in the energy autonomy, robots will need to extract energy from the environment. In many ways robots will face the same problems as animals [14]. We have added that an important variable is the state conditions in combination with environment events, because they may determine the system behavior.

In this way, a system must therefore have knowledge of its available resources as well as its components, their desired performance characteristics and their current status. Dynamic Power Management (DPM) is a design methodology for dynamically reconfiguring systems to provide the requested services and performance levels with a minimum number of active components or a minimum load on such components. It encompasses a set of techniques that achieves energyefficient computation by selectively turning off (or reducing the performance of) system components when they are idle (or partially unexploited) [16]. An autonomous robot planning tasks must be aware of power resources available [14]. Furthermore, low-power consumption is required to achieve acceptable autonomy in battery-powered systems, as well as to reduce the environmental impact (e.g., heat dissipation, cooling-induced noise) and operation cost of stationary systems [16].

Moreover, most electronic circuits and system designs are confronted with the problem of delivering high performance with a limited consumption of electric power, and achieving highly energy-efficient computation is a major challenge in electronic design [16]. In this context, a DPM and Real-Time Scheduling (RTS) techniques were presented in [17]. They were applied to reduce the power consumption of mobile robots. At the same time that scheduling is a key concept in computer multitasking and real-time operating system, the DPM dynamically adjusts power states of components adaptive to the task's need, reducing the power consumption without compromising system performance.

Mobile robots usually have multiple components, such as motors, microcontrollers and embedded computers. Whereas sensors collect data from environment, DC motors transform direct current into mechanical energy and are often used to drive the robots. In this model, the microcontrollers are used for low-level controls, whilst embedded computers for highlevel computation, providing an application programming interface (API). This high-level computation includes motion planning, image processing, and scheduling [17].

Finally, a case study of mobile robot's energy consumption and conservation shown that motion accounts for less than $50 \%$ of the total power consumption [17]. The power consumption of the microcontroller was very stable. This implies that other power consumers like computation have a big impact on power consumption [14]. These values were estimated by dividing the battery capacity by the time the computer can run with a fully charged battery when running different programs [17].

\section{A. Sensor Perception}

The perception of the environment is a major issue in autonomous vehicles. It uses many types of sensors [3], [4], including ultrasonic sensors, laser rangefinders, radar, cameras, etc. When incorporating several types of sensors, there is an increase of autonomy and "intelligence" degrees, especially in relation to navigation in unknown environments. In contrast, the type and quantity of sensors determine the volume of data for processing that requires, in most cases, a high computational cost. For unstructured environments, the scenario for study is dynamic, with several elements in motion. Thus, running an autonomous or semiautonomous system involves carrying out complex, and nondeterministic operations in real time.

A real-time system must satisfy explicit response-time constraints, including failure. This system is one whose logical correctness is based on both the correctness of the outputs and their timeliness [18]. Moreover, there is a considerable complexity in the sense that correctness not only depends on the logical ordering of events of the systems, but also on the relative timing between them [11].

Aware that in the majority of the autonomous and semiautonomous navigation systems, the machine-vision system is working together with other sensors, added to its low cost, in this work we have decided to use a monocular visionbased sensor. Because it uses simple techniques and fast algorithms, the system is capable to achieve a good performance, where the compromise between processing time and images acquisition is fundamental.

Additionally, the vision-based sensors are defined as passive sensors and the image scanning is performed fast enough for Intelligent Transportation Systems [19]. Furthermore, on the safety front, the progressive safety systems will be developed through the manufacturing of an "intelligent bumper" peripheral to the vehicle in answering new features as: blind spot detection, frontal and lateral precrash, etc. The objective in terms of cost to fill ADAS functions has to be very lower than the current Adaptive Cruise Control (500 Euros) [20].

\section{B. Machine Vision}

The machine vision (part of the embedded computers) is an important component of the set of sensors. Although extremely complex and highly demanding, thanks to the great deal of information it can deliver, it is a powerful means for sensing the environment and it has been widely employed to deal with a large number of tasks in the automotive field [19]. However, complex machine vision systems can lead to some losses due to the processing time. Thinking about the existing relation between a real-time decision system and a camera acquisition system that operates in a specific acquisition rate, the decision for a more complex machine vision system possibly leads to an excessively slow system for an independent real time application. The great amount of information would not 
necessarily lead to better decisions and could also harm the performance of the system, overloading it.

Taking into account that it has been estimated that humans perceive visually about $90 \%$ of the environment information required for driving [19], it is not a bad idea to reduce information acquired by a vision system, in order to reduce processing time. But the definition of an automatic image discarding criteria, which leads to a minimum loss of information, may not be a trivial task for computational systems, specially real-time ones. Then, based on the idea to reduce information acquired and in order to reduce processing time, we have proposed an automatic image discarding criteria [21], [22], based on Pearson's Correlation Coefficient (PCC) [15], a low complexity and easy implemented solution. It improves the performance of a realtime system by choosing, in an automatic way, which images should be discarded and which ones should be treated at the visual perception system. The inclusion of an automatic image discarding method leads in a reduction of the processing time. Although the system spends some milliseconds computing the PCC, it gains much more time discarding more than $90 \%$ of the images [22]. However, it is important to notice that this percentage is not dependent on the video sequence or image size, but on the obstacles / objects influence.

Furthermore, the fundamental premise for the applicability of DPM is that systems experience nonuniform workloads during operation time. A second assumption is that it is possible to predict, with a certain degree of confidence, the fluctuations of workload [16]. In this case, a simple DPM method shuts down a component when it is idle and it is essentially a prediction problem [17]. Additionally, in most real-world systems, there is little knowledge of future input events and DPM decisions have to be taken based on uncertain predictions. Thus, according to [16], the rationale in all predictive techniques is that of exploiting the correlation between the past history of the workload and its near future in order to make reliable predictions about future events. Moreover, workload observation and prediction should not consume significant energy.

Therefore, taking into account the temporal coherence between consecutive frames of a video sequence, we propose a new DPM methodology applied to a robotic visual machine perception. It also includes a cumulative impact data management.

\section{PeARSON's CoRrelation COEFFiCIENT (PCC)}

According to [23], an empirical and theoretical development that defined regression and correlation as statistical topics were presented by Sir Francis Galton in 1885. In 1895, Karl Pearson published the Pearson's Correlation Coefficient (PCC) [15]. The Pearson's method is widely used in statistical analysis, pattern recognition and image processing. Applications on the latter include comparing two images for image registration purposes, disparity measurement, etc [24]. It is described by Eq. (1):

$$
r_{1}=\frac{\sum_{i}\left(x_{i}-x_{m}\right)\left(y_{i}-y_{m}\right)}{\sqrt{\sum_{i}\left(x_{i}-x_{m}\right)^{2}} \sqrt{\sum_{i}\left(y_{i}-y_{m}\right)^{2}}}
$$

where $x_{i}$ is the intensity of the $i^{\text {th }}$ pixel in image $1, y_{i}$ is the intensity of the $i^{\text {th }}$ pixel in image 2, $x_{m}$ is the mean intensity of image 1 , and $y_{m}$ is the mean intensity of image 2 . The PCC threshold, $r_{1}$, has value 1 if the two images are identical, 0 if they are completely uncorrelated, and -1 if they are completely anti-correlated, for example, if one image is the negative of the other.

\section{DISCARDING CRITERIA}

The discarding criteria was presented in [21] as a simple solution to improve the performance of a real-time navigation system by choosing, in an automatic way, which images to discard and which ones to segment, in order to identify the navigation area. It was a new approach to the Pearson's Correlation Coefficient (PCC).

In Fig. 1, basically, if the PCC indicates that there is a high correlation between a reference frame and another new frame acquired, the new frame is discarded without being processed. In this case, some logical components may be shut down to save processor energy consumption, and/or to make the CPU available for running concurrent processes (the system can repeat a last valid command). Otherwise, the frame is processed and it is set as the new reference frame for the subsequent frame.

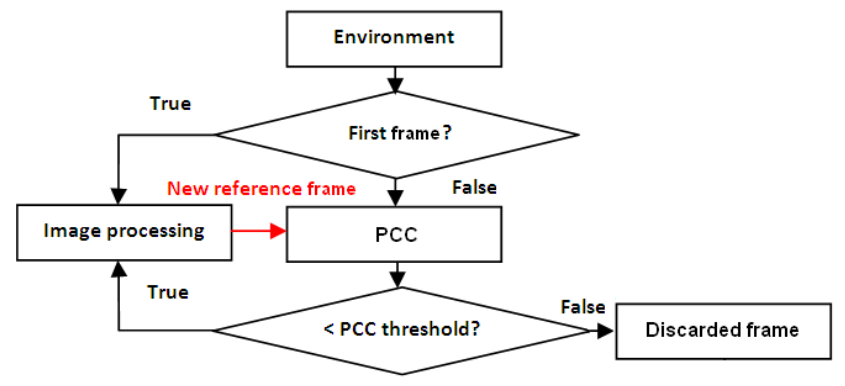

Fig. 1. Discarding criteria [21].

\section{LOGICAL DYNAMIC OPTIMIZATION}

According to [20], the road safety application can be important for other sectors such as aeronautics (for example guidance on the ground of planes), the railway, the sectors of security and defense, the property of equipment, etc. In this context, the fusion of sensors will allow to have a higher level of information and to get more various functions. However, it is important to consider all components to achieve better energy efficiency [17]. Moreover, an important variable is the state conditions in combination with environment events.

In this section, we introduce a logical dynamic optimization methodology. Based on the PCC variation and 
by exploiting the temporal coherence between consecutive frames, we propose a new environment observer method based on Pearson's method. Our monocular-vision system observes if there are no significant changes in the environment, permitting that some logical components may be shut down to save processor energy consumption, and/or to make the CPU available for running concurrent processes.

In this way, the robot can have many periodic tasks, such as motor and sensor control, sensing data reading, motion planning, and data processing. The robot may also have some aperiodic tasks, such as obstacle avoidance and communication. Furthermore, for mobile robots, the tasks' deadlines are different at different traveling speeds. At a higher speed, the periodic tasks have shorter periods [17].

Then, it is important to notice that there is no diffeomorphism between the vehicle speed and the PCC variation, because if there are no changes between consecutive frames, the PCC threshold remains static. In this case, the isomorphism cannot be guaranteed and it ensures more efficiency for our proposal.

The Fig. 2 (a) shows an autonomous displacement through the Mojave Desert [25], where the robot Stanley has used an average speed of $30.7 \mathrm{~km} / \mathrm{h}$ [26]. In Fig. 2 (b), due to PCC nature, taking a reference frame, in this case, the first frame of the Fig. 2 (a), a lower value of correlation is achieved when it is closer to the vehicle. That is, when the derivative approaches its maximum point, there is the obstacle detection. The Fig. 3 (a) shows the same case from a different representation. From an empirical PCC threshold equal to 0.85 (green line), the reference frames (red points) are closer when it is near to an obstacle. Above of the green line we have all discarded images. In Fig. 4 we also present the discarding rate for an off-road context.
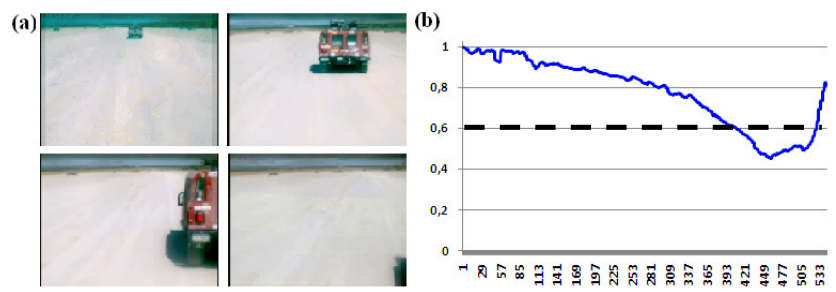

Fig. 2. (a): the frames of the desert video [25]; (b) From a reference frame, its correlation with all others; Blue line: the Pearson's correlation by Eq. (1).

Whereas the main problem that has to be faced when realtime imaging is concerned and which is intrinsic to the processing of images is the large amount of data [19], the Fig. 5 presents the accumulated time of a hypothetical image processing time $(15 \mathrm{~ms})$ versus the gain obtained by using the discarding criteria, which could allow significant savings in CPU power consumption. As shown in Fig. 3, in desert context were discarded 470 of 530 frames, whilst in off-road context were discarded 5595 of 6740 frames. For these two cases, the discarding rate remains over $80 \%$.

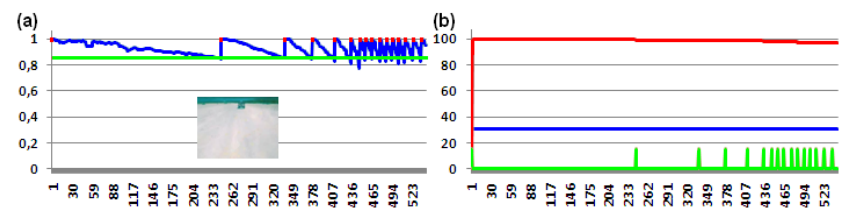

Fig. 3. Desert video [25]: (a) Green line: empirical PCC threshold equal to 0.85; Above of the green line we have the discarded images; Red points: reference frames; (b) Red line: discarding rate; Blue line: vehicle speed; Green line: hypothetical image processing time $(15 \mathrm{~ms})$.

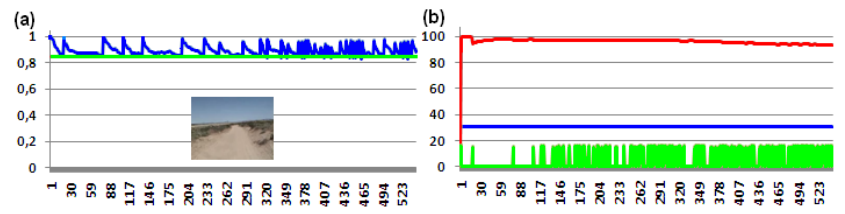

Fig. 4. Part of the off-road video [25]: (a) Green line: empirical PCC threshold equal to 0.85; Above of the green line we have the discarded images; (b) Red line: discarding rate; Blue line: vehicle speed; Green line: hypothetical image processing time (15ms).
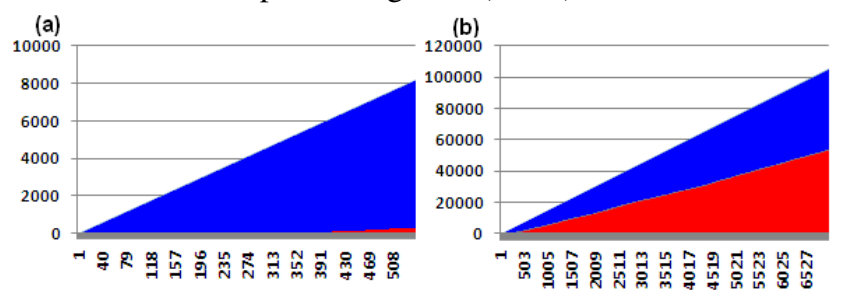

Fig. 5. (a) Desert video [25]; (b) Off-road video [25]; In blue: the cumulative impact computations (ms) without the discarding criteria; In red: the cumulative computations (ms) by using the discarding criteria.

\section{A. Cumulative Impact Data Management (CIDM)}

Existing studies on energy reduction for robots focus on motion planning to reduce motion power. However, other components like sensing, control, communication and computation also consume significant amounts of power [17]. According to the Pearson's correlation, in a certain analysis window (pair of frames), if the obstacle/object occupies a big portion of the scene, the PCC threshold tends to be low. Conversely, if obstacle/object occupies a small portion of the frame, it means that it is away from the vehicle and the system will have time enough to react. Nevertheless, where are these interest points/pixels? Or, which pixels (interest points) of the pair of images contributed most to the Pearson's coefficient computed? Which of them really need to be reprocessed (or resent to a server)?

Right after the Pearson's correlation in Eq. (1), we have $x_{m}$ and $y_{m}$, respectively: the mean intensities of images 1 and 2 . From these values, we begin again the process's correlation by applying,

$$
r_{2}=\frac{\sum_{i}\left(x_{i}-r_{1 X m}\right)\left(y_{i}-r_{1 Y m}\right)}{\sqrt{\sum_{i}\left(x_{i}-r_{1 X m}\right)^{2}} \sqrt{\sum_{i}\left(y_{i}-r_{1 Y m}\right)^{2}}}=\left\{\begin{array}{l}
-1 \\
o r \\
+1
\end{array}\right.
$$


where $x_{i}$ is the intensity of the $i^{\text {th }}$ pixel in image $1, y_{i}$ is the intensity of the $i^{\text {th }}$ pixel in image 2, $r_{1 X m}$ and $r_{1 Y m}$ were obtained in Eq. (1).

For each pair of pixels analyzed in Eq. (2), the only possible result is: $[-1$ or +1$]$. That is, all pixels with intensities below these means will be candidates for interest points. Fig. 6 (c) and (f) present the CIDM process, where the red pixels (interest points) represent $r_{2}=-1$.

As shown in Fig. $6(\mathrm{~g})$, by processing only when $r_{2}=-1$, in desert video were processed about 205 thousand points, instead of 3.7 million points. As shown in Fig. 6 (h), in offroad context were processed about 10 million points, instead of 48 million points. In blue, the cumulative impact data without the discarding criteria. In red, the cumulative data by using the discarding criteria.

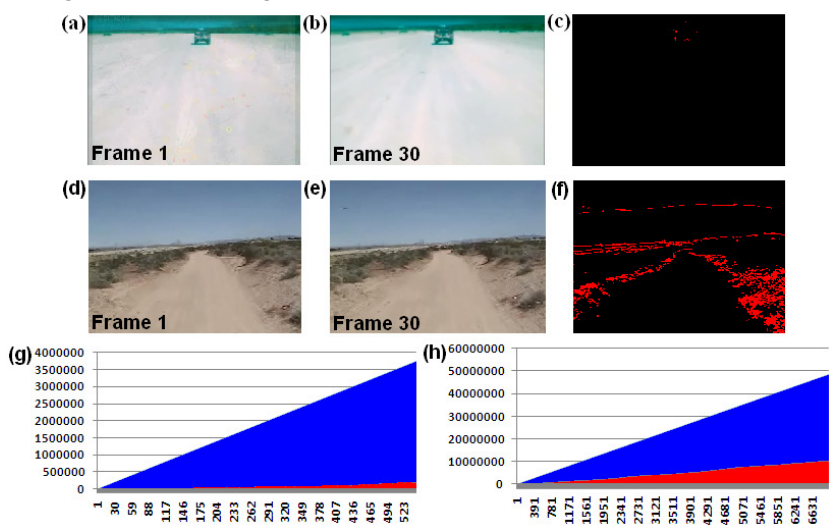

Fig. 6. (a) and (b) are frames of the desert video [25]; (d) and (e) are frames of the off-road video [25]; (c) and (f) present the process's correlation by Eq. (2), where the red pixels (interest points) represent $r_{2}=-1$; (g) and (h) represent the cumulative impact data.

\section{EXPERIMENTAL RESULTS}

As has been shown above in Section V, the Fig. 7, 8 and 9 show the performance of our proposed method in real, dynamic and unknown environments. For all these cases, the discarding rate remains over $65 \%$.

These results were obtained in real conditions using our experimental vehicle. As illustrated in Fig. 10, the vehicle was equipped with a camera to acquire $320 \times 240$ color images at $20 \mathrm{ftps}$, and a CAN-bus gateway provides the speed of the rear-wheels (WSS) and the yaw rate of the vehicle (from the ESP). It was tested on a $2.5 \mathrm{GHz}$ Intel Core 2 Quad processor, 3.48 GB RAM, Microsoft Windows XP Professional SP3, Visual Studio C++ and OpenCV 2.1.0. In order to reduce the number of data, it also includes the resolution reduction of image (to $96 \times 72$ ).

Fig. 9 (a) presents the computational mean time of a horizon finding algorithm [27] in unknown and urban environment. In this way, from an empirical PCC threshold equal to 0.85 , the red line shows that the computational mean time was $196 \mathrm{~Hz}(5.09 \mathrm{~ms})$, against $64 \mathrm{~Hz}(15.62 \mathrm{~ms})$ without the discarding criteria. In Fig. 9 (b), above the green line, we have the discarded images.

For additional results see [28].

(a)

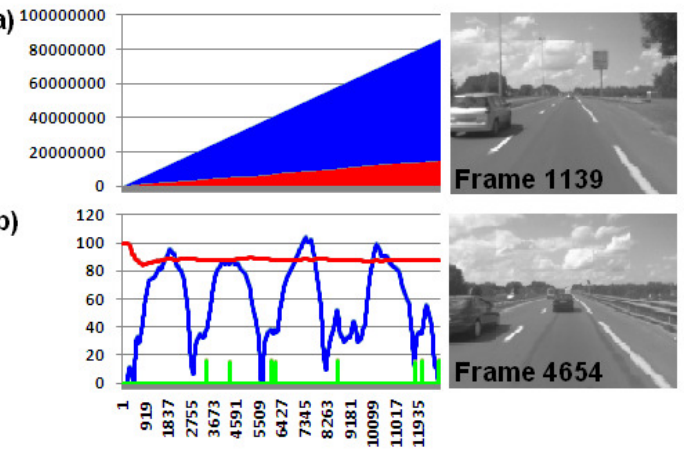

Fig. 7. Real environment: (a) The CIDM performance; (b) Red line: discarding rate; Blue line: vehicle speed; Green line: computational time (ms).

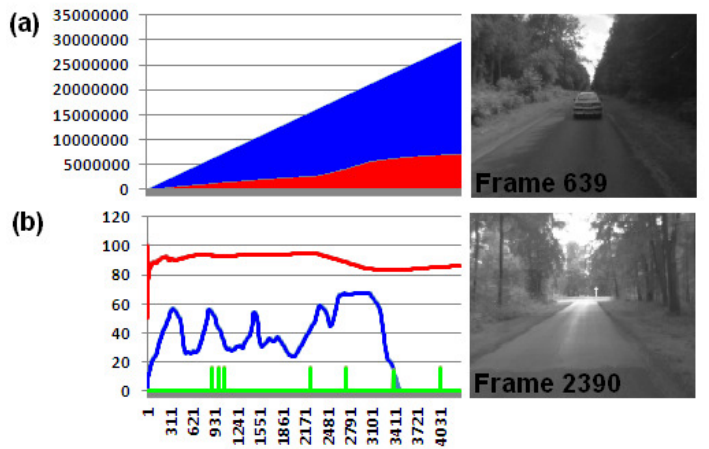

Fig. 8. Real environment: (a) The CIDM performance; (b) Red line: discarding rate; Blue line: vehicle speed; Green line: computational time (ms).

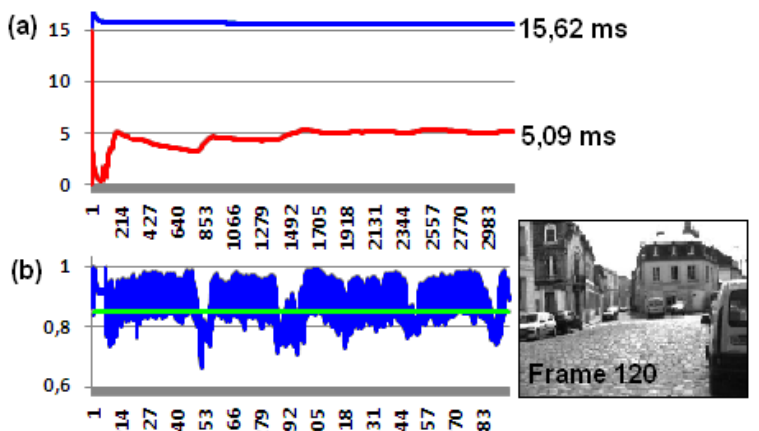

Fig. 9. The computational mean time in urban environment.

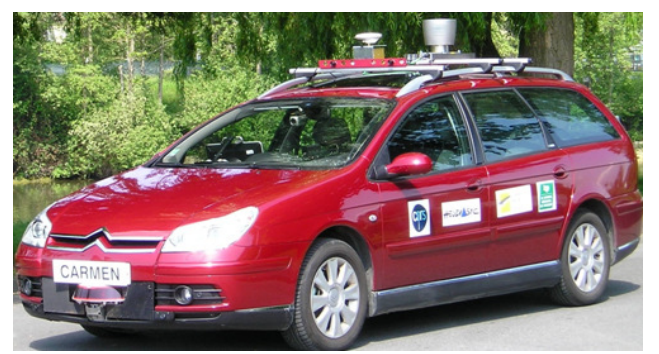

Fig. 10. The experimental vehicle laboratory with the monocular vision system. 


\section{CONCLUSION}

We have proposed a new environment observer method based on Pearson's Correlation Coefficient. We are implementing this DPM methodology in a real experimental test-bank, to evaluate, in real time, the real energy consumption economy in terms of electrical current used by the visual machine. A remarkable characteristic of methodology presented in this work is its independence of the image acquiring system and of the robot itself. The same implementation works in different mobile robots, with different embedded vision systems, without the need of adjusting parameters. Moreover, because there is no diffeomorphism between the vehicle speed and the PCC variation, it ensures more efficiency for our proposal. Nevertheless, this visual-observer methodology may be extended to other sensors and components.

\section{ACKNOWLEDGMENT}

The authors wish to thank Mr. Gerald Dherbomez for his support in data acquisition. This work was supported in part by French National Research Agency / ANR PERCOIVE Project.

\section{REFERENCES}

[1] B. Kim, P. Hubbard, D. Necsulescu, (2003), "Swarming Unmanned Aerial Vehicles: Concept Development and Experimentation, A State of the Art Review on Flight and Mission Control", DRDC-OTTAWA-TM-2003-176; Technical Memorandum.

[2] F. Bonin-Font, A. Ortiz, G. Oliver, (2008), "Visual Navigation for Mobile Robots: A Survey", Journal of Intelligent and Robotic Systems.

[3] S. Thrun, et al. (2006), "Stanley, the robot that won the DARPA Grand Challenge", Journal of Robotic Systems, Volume 23, Issue 9, DARPA Grand Challenge, 661-692.

[4] Team Berlin (2007), Spirit of Berlin: An Autonomous Car for the DARPA Urban Challenge Hardware and Software Architecture, http://www.darpa.mil/grandchallenge/ TechPapers/Team_Berlin.pdf [retrieved 02 Dec. 2010].

[5] O. Gietelink, J. Ploeg, B. De Schutter, and M. Verhaegen, (2006) "Development of advanced driver assistance systems with vehicle hardware-in-the-loop simulations," Vehicle System Dynamics, vol. 44, no. 7, pp. 569-590.

[6] S.R. Anton, D.J. Inman, (2008), "Energy Harvesting for Unmanned Aerial Vehicles", In: Proceeding of SPIE.

[7] A. T. Klesh and P. T. Kabamba, (2009), "Solar-Powered Aircraft: Energy-Optimal Path Planning and Perpetual Endurance", Journal of Guidance, Control, and Dynamics.

[8] The Vision for Space Exploration, NASA, Feb. 2004, http://www.nasa.gov/pdf/55583main_vision_space_exploratio n2.pdf [retrieved 29 Nov. 2010].

[9] Energetically Autonomous Tactical Robot, (2009), DARPA Contract W31P4Q-08-C-0292.

[10] R. Finkelstein, Robotic Technology Inc, (2009), "Energetically Autonomous Tactical Robot and Associated Methodology of Operation", Patent Application number: 12/612,489, Publication number: US 2010/0155156 A1.

[11] P. Bouyer et al., (2010), "Quantitative analysis of real-time systems". Journal Communications of the ACM.

[12] K. Obayashi and K. Tani, (2004), "Concept of Vehicle
Electric Power Flow Management System (VEF)", SAE 2004 World Congress \& Exhibition No.2004-01-0361, Detroit, MIl, USA.

[13] K. Morita and K. Shimamura, (2003), "Examination of the Evaluation Methods for Hybrid Electric Vehicles", Japan Automobile Research Institute Research Journal, vol.25, No. 1, pp. 9-12.

[14] A. Deshmukh, P. A. Vargas, R. Aylett and K. Brown, (2010), "Towards Socially Constrained Power Management for LongTerm Operation of Mobile Robots", 11th Conference Towards Autonomous Robotic Systems, Plymouth, UK.

[15] K. Pearson, (1895), Royal Society Proceedings, 58, 241.

[16] L. Benini, A. Bogliolo, and G. D. Micheli. (2000), "A Survey of Design Techniques for System-Level Dynamic Power Management", IEEE Transactions on Very Large Scale Integration Systems, 8(3):299-316.

[17] H. Y. L. C. Yongguo Mei, Yung-Hsiang Lu, (2005), “A case study of mobile robot's energy consumption and conservation techniques", in 12th IEEE ICAR, pp. 492--497.

[18] P. Laplant, (2004), "Real-Time System Design and Analysis", IEEE Press, 3nd ed.

[19] M. Bertozzi. A. Broggi and A. Fascioli, (2000), "Vision-based intelligent vehicles: state of the art and perspectives", Robotics and Autonomous systems 32, 1-16.

[20] Radio Spectrum Committee, European Commission, Public Document, Brussels, 5 July 2010, RSCOM10-35 http://ec.europa.eu/information_society/policy/ecomm/radio_s pectrum/_document_storage/rsc/rsc32_public_docs/rscom10_ 35.pdf [retrieved 02 Dec. 2010]

[21] A. Miranda Neto, L. Rittner, N. Leite, D. E. Zampieri, R. Lotufo and A. Mendeleck, (2007), "Pearson's Correlation Coefficient for Discarding Redundant Information in Real Time Autonomous Navigation Systems", Proceedings of the 2007 IEEE Multi-conference on Systems and Control.

[22] A. Miranda Neto, L. Rittner, N, Leite, D. E. Zampieri and A. C. Victorino, (2008), "Nondeterministic Criteria to Discard Redundant Information in Real Time Autonomous Navigation Systems based on Monocular Vision”, ISIC Invited Paper, 2008 IEEE Multi-conference on Systems and Control, San Antonio, Texas, EUA, September 3-5.

[23] J. L. Rodgers and W. A. Nicewander, (1988), "Thirteen Ways to Look at the Correlation Coefficient", The American Statistician, 42, 59-66.

[24] Y. K. Eugene and R.G. Johnston, "The Ineffectiveness of the Correlation Coefficient for Image Comparisons", Technical Report LA-UR-96-2474, Los Alamos, 1996.

[25] DARPA 2005. "DARPA Grand Challenge", http://www.darpa.mil/grandchallenge05/

[26] Stanford Racing Team's Entry In The 2005 DARPA Grand Challenge, (2006, June 10), http://www.stanfordracing.org/

[27] A. Miranda Neto, A. C. Victorino, I. Fantoni and D. E. Zampieri, (2011), "Robust Horizon Finding Algorithm for Real Time Autonomous Navigation based on Monocular Vision", in reviewing process. The video can be accessed at http://www.youtube.com/watch?v=8KbZ1J0txUE

[28] http://www.youtube.com/watch?v=XaZndmMaieE 